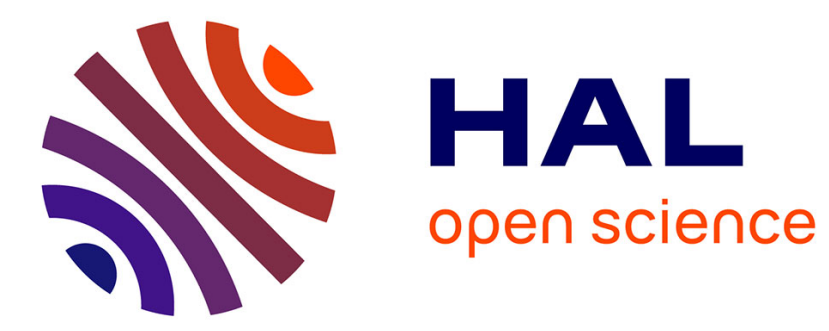

\title{
Compressive sensing in medical ultrasound
}

\author{
H. Liebgott, A. Basarab, Denis Kouamé, Olivier Bernard
}

\section{To cite this version:}

H. Liebgott, A. Basarab, Denis Kouamé, Olivier Bernard. Compressive sensing in medical ultrasound. IEEE International Ultrasonics Symposium 2012, IEEE, Oct 2012, Dresden, Germany. pp.1-6, 10.1109/ULTSYM.2012.0486 . hal-00830731

\section{HAL Id: hal-00830731 \\ https://hal.science/hal-00830731}

Submitted on 8 Jan 2021

HAL is a multi-disciplinary open access archive for the deposit and dissemination of scientific research documents, whether they are published or not. The documents may come from teaching and research institutions in France or abroad, or from public or private research centers.
L'archive ouverte pluridisciplinaire HAL, est destinée au dépôt et à la diffusion de documents scientifiques de niveau recherche, publiés ou non, émanant des établissements d'enseignement et de recherche français ou étrangers, des laboratoires publics ou privés. 


\title{
Compressive sensing in medical ultrasound
}

\author{
${ }^{1}$ Hervé Liebgott, ${ }^{2}$ Adrian Basarab, ${ }^{2}$ Denis Kouame, ${ }^{1}$ Olivier Bernard and ${ }^{1}$ Denis Friboulet
}

\author{
${ }^{1}$ Université de Lyon, CREATIS, \\ CNRS UMR 5220, INSERM U630, Université de Lyon, \\ ${ }^{1}$ Université Lyon $1,{ }^{2}$ INSA Lyon \\ Lyon, France \\ e-mail: name@,creatis.insa-lyon.fr
}

\author{
${ }^{2}$ Université de Toulouse, IRIT \\ CNRS UMR 5505, Université Paul Sabatier \\ Toulouse, France \\ e-mail: namet@,irit.fr
}

\begin{abstract}
One of the fundamental theorem in information theory is the so-called sampling theorem also known as Shannon-Nyquist theorem. This theorem aims at giving the minimal frequency needed to sample and reconstruct perfectly an analog band-limited signal. Compressive sensing (or compressed sensing, compressive sampling) or $\mathrm{CS}$ in short is a recent theory that allows, if the signal to be reconstructed satisfies a number of conditions, to decrease the amount of data needed to reconstruct the signal. As a result this theory can be used for at least two purposes: i) accelerate the acquisition rate without decreasing the reconstructed signal quality (e.g. in terms of resolution, SNR, contrast ...) ii) improve the image quality without increasing the quantity of needed data.
\end{abstract}

Even if medical ultrasound is a domain where several potential applications can be highlighted, the use of this theory in this domain is extremely recent.

In this paper we review the basic theory of compressive sensing. Then, a review of the existing CS studies in the field of medical ultrasound is given: reconstruction of sparse scattering maps, pre-beamforming channel data, post-beamforming signals and slow time Doppler data.

Finally the open problems and challenges to be tackled in order to make the application of CS to medical US a reality will be given.

Keywords: compressive sensing, sparse, beamforming, ultrasound

\section{INTRODUCTION}

Whereas the Shannon theorem fixes the limit for the sampling frequency of a signal to twice its maximum frequency component $f_{\max }$, the recently introduced compressed sensing (CS) theory allows - under certain assumptions - to recover a signal sampled far below this limit [1-3]. Compressed sensing (also known as compressive sensing or compressive sampling) can be applied for two main purposes. i) it can lower the amount of data needed and thus allows to speed up acquisition. An example in the field of medical imaging of such application is dynamic MRI [4] ii) it can improve the reconstruction of signals/images in fields where constraints on the physical acquisition set up yields very sparse data sets. A typical example is seismic data recovery in geophysics [5].

The application of this theory to the field of medical ultrasound is extremely recent even though there are applications that are excellent candidates such as e.g. triplex acquisitions [6] for $\mathrm{CFM} / \mathrm{B}$ mode/Doppler or 3D imaging using matrix arrays. The objective of this paper is to give the reader an overview of the different attempts to show the feasibility of CS in medical ultrasound. The classification of the studies is done according to the data that are considered to be sparse (the scatterer distribution itself, the pre-beamforming channel data, the beamformed RF signal and even Dopple data). For each example the potential of using CS is highlighted as well as the specificity in terms of modeling or numerical implementation.

The paper is organized as follows: in section II the CS theory is recalled, section III presents different applications of CS in medical ultrasound and finally section IV gives a short discussion and conclusion

\section{COMPRESSIVE SENSING THEORY}

Compressive sensing (CS) [2] allows the reconstruction of a signal $\mathbf{x} \in \mathbb{R}^{n}$ from a linear combination of a small number of random measurements $\mathbf{y} \in \mathbb{R}^{m} \mathrm{~m}<\mathrm{n}$.

In a general setting, the measurements $\mathbf{y}$ may be acquired in the so-called "sensing basis" $\Phi$, which depends on the acquisition device. As an example, in MRI, $\Phi$ is the Fourier basis.

$$
\mathbf{y}=\Phi \mathbf{x}
$$

where $\Phi$ is an $\mathrm{m} \times \mathrm{n}$ matrix. The columns of $\Phi$ have an entry one at random positions and zero elsewhere, thereby modeling the random selection of the measurements.

At the heart of CS is the assumption that $\mathbf{x}$ has a sparse representation in some model orthonormal basis $\Psi$, i.e.:

$$
\mathbf{x}=\Psi \mathbf{v}
$$


where $\mathbf{v}$ has only $\mathrm{s}<\mathrm{m}<\mathrm{n}$ non zero coefficients. The signal $\mathbf{v}$ is called s-sparse. CS theory shows that this sparsity allows an exact recovering of $\mathbf{v}$ with overwhelming probability for a certain class of matrices $\Phi \Psi$ [1]. In particular, the sensing basis has to be incoherent with the model basis $\Psi$ [7], which is ensured by the randomness of the non-zeros components of $\Phi$. Finally the problem can be written as follows:

$$
\mathbf{y}=\Phi \Psi \mathbf{v}=\mathbf{A v}
$$

where $\mathbf{A}$ is an $\mathrm{m} \times \mathrm{n}$ full rank matrix (i. e. the $\mathrm{m}$ raws of $\mathbf{A}$ are independent).

In these settings, the CS problem thus amounts to solve (3) for $\mathbf{v}$, under the constraint that $\mathbf{v}$ is sparse. Once $\mathbf{v}$ is estimated, the signal $\mathbf{x}$, can then be computed from (2).

For matrices A with a specified isometry constant of the socalled "restricted isometry property" (RIP), Candès et al. [1] showed that the CS problem may be solved through the following $\ell_{0}$-minimization problem $\mathrm{P} 0$ :

$$
\text { P0 } \quad \hat{\mathbf{v}}=\underset{\mathbf{v} \in \mathbb{R}^{n}}{\arg \min }\|\mathbf{v}\|_{\ell_{0}} \text { subject to } \mathbf{y}=\mathbf{A v}
$$

where the $\ell_{0}$ norm is $\|\mathbf{v}\|_{\ell_{0}}:=\left|\left\{i, v_{i} \neq 0\right\}\right|$

Equation (4) thus implies that from all the possible solutions of (3), we seek the sparsest one. In general, solving (4) is NP hard. Sub-optimal greedy algorithm attempt to solve this problem by successively adding non zero components to a sparse approximation of $\mathbf{v}$. (see [8]).

By imposing a more restrictive bounds on the isometry constant, the sparsest solution $\hat{\boldsymbol{x}}$ of (3) can be found by solving the following basis pursuit (BP) problem P1 [9, 10]:

$$
\text { P1 } \quad \hat{\mathbf{v}}=\underset{\mathbf{v} \in \mathbb{R}^{n}}{\arg \min }\|\mathbf{v}\|_{\ell_{1}} \text { subject to } \mathbf{y}=\mathbf{A v}
$$

where the $\ell_{1}$ norm is $\|\mathbf{v}\|_{\ell_{1}}=\sum_{i=1}^{n}\left|v_{i}\right|$.

The $\ell_{0}-\ell_{1}$ equivalence, using the RIP, was presented by Candès in [9] (see also [1]).

The framework described above assumes that we are given exact samples of the signal to be recovered. This is seldom the case in practice, since the measurements are very often corrupted by noise. In the case of measurements with additive noise, we have:

$$
\mathbf{y}=\mathbf{A v}+\mathbf{e}
$$

where e represent a noise term of bounded energy $\|\mathbf{e}\|_{\ell_{2}} \leq \varepsilon$, $\mathrm{P} 1$ can be then recast as $[9,11]$ :

$$
\text { P2 } \quad \hat{\mathbf{v}}=\underset{\mathbf{v} \in \mathbb{R}^{n}}{\arg \min }\|\mathbf{v}\|_{\ell_{1}} \text { subject to } \quad \mathbf{y}=\|\mathbf{y}-\mathbf{A x}\|_{\ell_{2}} \leq \varepsilon
$$

In practical applications the signal is generally not exactly sparse but most of its coefficients in (2) are small. When signal coefficients $\mathbf{v}$ decays exponentially in absolute value, the signal is called 'compressible'. The solution found by P1 (5) or P2 (7) gives the approximation of $\mathbf{v}$ by keeping its $\mathrm{S}$ largest entries.

For solving P1 and P2, mainly two classes of methods have been employed. The first one consists in deterministic optimization algorithms. The second one uses stochastic (Bayesian) algorithms. With this approach, statistical a priori laws are associated to each of the unknown variables, further combined using the Bayes law to find the a posteriori probability to be maximized.

$$
p(\boldsymbol{v} \mid \boldsymbol{y}) \propto p(\boldsymbol{y} \mid \boldsymbol{v}) p(\boldsymbol{v})
$$

In (8), $p(\boldsymbol{y} \mid \boldsymbol{v})$ represents the likelihood and $p(\boldsymbol{v})$ contains prior information about the unknown $\boldsymbol{v}$. Note that if the noise is considered Gaussian and the prior law on $\boldsymbol{v}$ is a Laplacian, the maximization of the posterior probability in (8) is equivalent to the classical LASSO optimization.

\section{APPLICATION TO ULTRASOUND IMAGING}

A central concern in CS is that the data under consideration should have sparse expansion in some dictionaries (e.g., Fourier basis, wavelet basis, dictionary learned from data, etc...), i.e., the number of non-zero coefficients of the image or signal in this representation basis should be as small as possible. Moreover, US echographic data may be considered at various stages of the image formation pipeline. In a conventional configuration, the set of raw radio-frequency (RF) signals received at each transducer element (i.e. channel RF data) is beamformed to build the beamformed RF image. Detection applied to the latter yields the envelope-detected image, and finally the log-envelope image corresponding (possibly after some interpolation) to the conventional B-mode display is obtained. As a consequence, one of the main features of the existing studies is the type of signal/image to be reconstructed and the choice of the representation where the US data are assumed to be sparse. Another important characteristic of these studies corresponds to the formalization of the reconstruction problem and related algorithm (e.g., Bayesian modeling, deterministic basis pursuit, or matching pursuit).

\section{A. Sparse diffusion map}

Several groups of authors [12-18] have chosen to model the medium under investigation itself as a sparse distribution of scatterers. The discrete scattering model is commonly used in the literature and is the basis for the most used ultrasound simulation program (Field II [19, 20]). However considering that most of the scatterers have an echogenecity close to zero is more unusual.

The approach proposed by $[12,13]$ aims at producing, with only one single plane wave transmission, an image having equivalent spatial resolutions as with conventional imaging. The potential of the approach is to provide fast imaging. Indeed in such a situation the highest possible temporal resolution (1 image per transmitted pulse) would be reached. The basic idea is to write the direct scattering problem and solve the inverse problem under the constraint that the scatterer distribution is sparse. This can be written as follows

$$
p^{s c}\left(e_{\theta}\right)=G\left(e_{\theta}\right) \gamma_{\kappa}
$$

with $p^{s c}\left(e_{\theta}\right)$ the scattered pressure received by the transducer elements after transmission of a plane wave in direction $\theta, G\left(e_{\theta}\right)$.represents propagation and interaction 
with the scatterers and $\gamma_{\kappa}$ is the scatterer distribution lying on a $\mathrm{N}_{\mathrm{z}}$ times $\mathrm{N}_{\mathrm{x}}$ regular grid. Solving the inverse scattering problem is an ill posed linear problem equivalent to the CS problem described in equation (4) or (5) if $\gamma_{\kappa}$ is assumed to be sparse. As a result it can be solved by $\ell_{1}$ minimization. Figure 1 shows a result from a simple phantom consisting in four isolated scatterers obtained with this approach. Here the sparsity assumption is obviously perfectly verified and the result outperforms even the synthetic aperture approach.

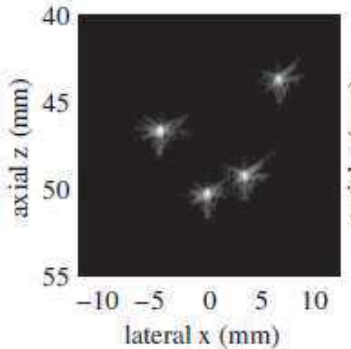

(a) SA

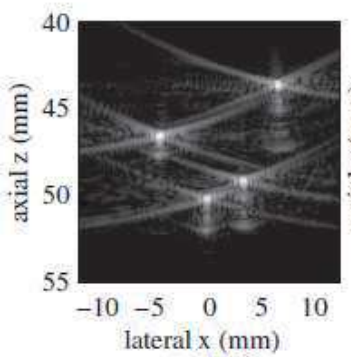

(c) FBP

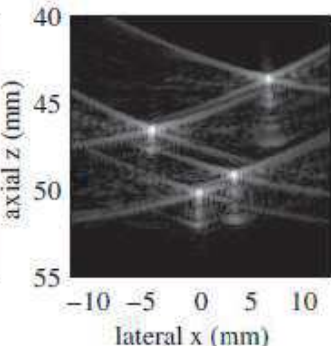

(b) DAS

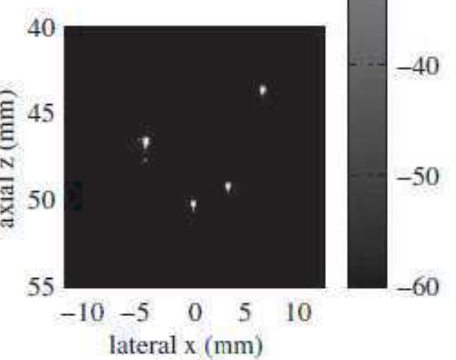

(d) CS
Figure 1. Result from Shiffner 2012 obtained from a phantom consisting in four isolated scatterers. The CS result (d) is compared with synthetic aperture (a), delay and sum (b) and Fourier back propagation (c)
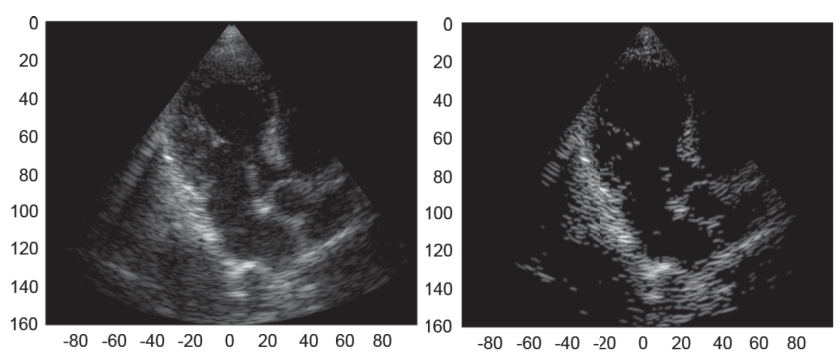

Figure 2. Result from Wagnerr 2012(a) original cardiac image (b) image obtained by CS. The edges are well reconstructed but the speckle is close to be completely lost

With the same assumption $[14,15]$ proposed another approach based on finite rate of innovation and Xampling. The technique is used to reconstruct cardiac images as reported in Figure 2. In these images the edges are clearly preserved whereas the speckle patterns are much reduced and even lost in some parts of the images. This is consistent with the assumption of scatterer map sparsity. Indeed such an hypothesis tends to keep only the highest echoes and to reduce the small ones which are however extremely important for the construction of the speckle patterns.

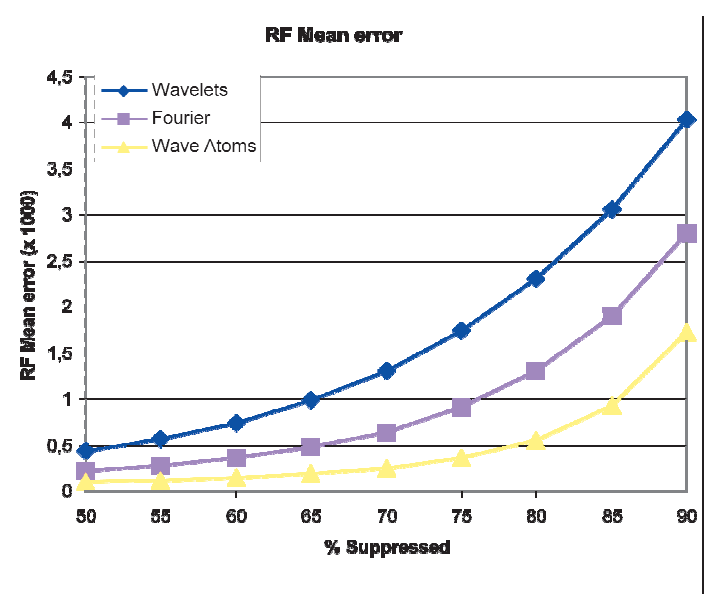

Figure 3. Result from [21] obtained from simulated data. The plot compares the reconstruction error as a function of removed quantity of data and sparsifying basis. Wave atoms lead to the best result

\section{B. Sparse Raw RF}

Another group of authors [21-24] consider that the raw channel data gathered at each transducer element during receive have a sparse decomposition in some basis. The objective of such an approach is to reduce the quantity of prebeamformed data acquired and evaluate the ability of this approach to reconstruct B-mode images of good quality. In [21] three sparsifying bases $\Psi$ have been tested: wavelets, Fourier and the recently introduced wave-atoms basis [25]. The acquisition of the raw data measurements was done using a spatially uniform random sampling. Reconstruction was done using $\ell_{1}$ minimisation. The study was carried out through simulations using Field II and experimental data acquired with the Ultrasonix MDP scanner.

Figure 3 shows a comparison of the mean reconstruction error as a function of removed data and sparsifying basis. As expected, for all bases the error of reconstruction increases when more data is removed. The most interesting thing here is that wave atoms lead to an error twice as small as Fourier and three times as small as wavelets.

In order to evaluate from a visual point of view the ability of this approach to reconstruct good quality B-mode images, Figure 4 shows experimental results obtained from only $20 \%$ of the original data using CS and wave atoms.

According to this result one might be extremely confident on the ability of CS to provide the right framework for ultrasound image reconstruction. However one important point that has not been mentioned here is the measurement setup. The obtained results indeed correspond to a spatially uniform random acquisition of the raw channel data-set. Acquiring the data this way is probably as difficult as just acquiring all the channel data set. An interesting approach would be to remove entire columns of the raw data set. This would indeed consist in disconnecting some elements of the probe. This could be extremely interesting for $3 \mathrm{D}$ imaging with matrix arrays in order to reduce the typical huge number of elements of such arrays. Unfortunately the initial results of this approach which are not given here show that this strategy leads to images of much lower quality. 

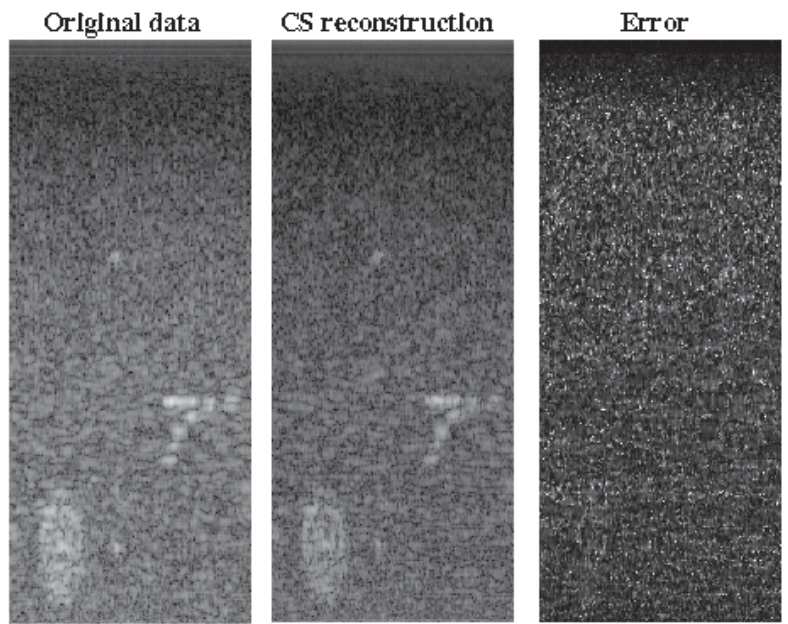

Figure 4. Experimental results from [21] showing the feasibility of the proposed technique to reconstruct pre-beamformed data able to lead to good quality B-mode images. The error is spread evenly over the entire image and both the strong structures and the speckle are reconstructed.

C. Sparse assumption of the RF images Fourier transform In this section the reconstruction of post-beamforming $2 \mathrm{D} \mathrm{RF}$ images via CS technique is discussed. The sparsity assumption is related here to the assumption of bandlimited RF signal acquisition. Thus, the 2D Fourier transform of RF images is assumed to be sparse. With the notations introduced in section II, $\Phi$ stands in this section for the spatial acquisition mask (showing the random positions where spatial samples are measured), $\Psi$ is the $2 \mathrm{D}$ inverse Fourier transform, $\boldsymbol{y}$ are the randomly measured post-beamformed RF samples and $v$ is the 2D Fourier transform of the RF image to be reconstructed. Thus, considering an additive Gaussian noise $\boldsymbol{\eta}$ with zero-mean and variance $\sigma^{2}$, the acquisition model is:

$$
y=A v+\eta
$$

With $\quad \boldsymbol{y}=\left(y_{1}, \ldots, y_{M}\right) \in \mathbb{C}^{M}, \quad \boldsymbol{v}=\left(v_{1}, \ldots, v_{N}\right) \in \mathbb{C}^{N}$ and $\boldsymbol{\eta}=\left(\eta_{1}, \ldots, \eta_{M}\right) \in \mathbb{C}^{M}$ representing the vectorized forms of the corresponding images.

From a theoretical point of view, the ideal sampling mask $\Phi$ (the most incoherent with the Fourier transform) is a uniform random pattern in the two spatial directions. As previously mentioned, it has however only a limited interest in US imaging. For this reason, a more suitable random sampling mask has been proposed in [26-28]. It considers that randomly chosen beamformed RF lines are not sampled at all, and is thus less incoherent with the Fourier transform. Thanks to this sampling scheme, the number of US pulses emitted during the acquisition is potentially reduced and as a consequence the acquisition time reduced. An example of such a 2D sampling mask is shown in Figure 5(for its extension in 3D, see [26, 28]).

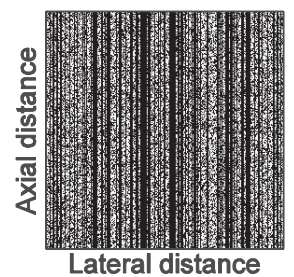

Figure 5. Random post-beamforming RF sampling mask. The white pixels correspond to the measured samples locations.

The resulting inverse problem in (10) can be solved using a conjugate gradient descent method, as shown by the results obtained in [28]. However, more accurate results are provided in $[29,30]$ using a Bayesian framework. We give hereafter the main details of the Bayesian model proposed in [30] and its extension to a regularized form in [29]. Given the inverse problem in (10) and keeping in mind that $\boldsymbol{\eta}$ is a zero-mean Gaussian noise, the associated likelihood is:

$$
p\left(\boldsymbol{y} \mid \boldsymbol{v}, \sigma^{2}\right)=\frac{1}{\left(\pi \sigma^{2}\right)^{N}} \exp \left(\frac{1}{\sigma^{2}}\|\boldsymbol{y}-A \boldsymbol{v}\|_{2}^{2}\right)
$$

Where $\|*\|_{2}$ stands for the standard $l_{2}$ norm.

At this stage, the inverse problem has two unknowns, the variance of the noise and $v$, the $2 \mathrm{D}$ Fourier transform of the RF image. For the latter, two assumptions are taken into account in order to construct the prior distribution: the Gaussian property of the RF images and image sparsity in the Fourier domain. Thus, each pixel $v_{i}$ of the Fourier domain has been assigned a mixture of a Gaussian statistical law and a mass at the origin.

$$
\begin{aligned}
p\left(v_{i} \mid w, \sigma_{v}^{2}\right)=(1 & -w) \delta\left(\left|v_{i}\right|\right) \\
& +\frac{w}{\pi \sigma_{v}^{2}} \exp \left(-\frac{\left|v_{i}\right|}{\sigma_{v}^{2}}\right)
\end{aligned}
$$

Where $w$ is the prior probability of having a non-zero in the Fourier domain. In [30], $w$ was considered a priori uniform random. In [29], assuming that the non-zero pixels in the Fourier domain of a RF image are located in compact regions around the central frequency of the probe, a further spatial regularization is taken into account by the prior on $w$.

Note that the Gaussian characteristics may be replaced by other laws reported in the literature. For example, in the context of the application of CS in US imaging, Achim et al. used in [31] an $\alpha$-stable distribution.

The posterior distribution is maximized using a MCMC method (also known as Gibbs sampler). The details concerning the sampling of the unknown variables over the iterations are given in $[29,30]$. We give hereafter a simulation result extracted from [29], showing the original simulated image, the samples used for reconstruction and the results obtained using a gradient descent algorithm and with the Bayesian framework. In this case, $10 \%$ of the original image pixels are used for reconstruction (30\% randomly located samples from $30 \%$ randomly chosen RF lines). 


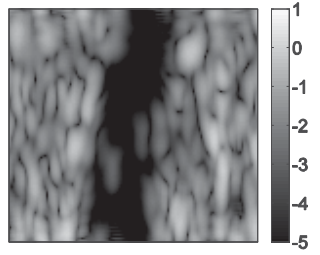

(a)

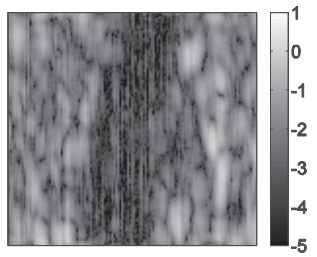

(c)

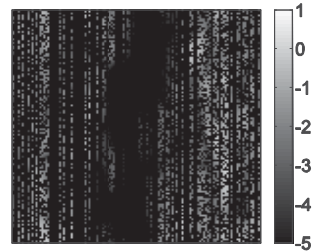

(b)

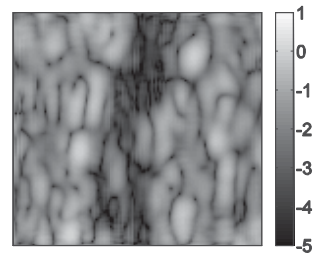

(d)
Figure 6. B-mode images corresponding to: (a) original simulated RF image, (b) RF samples used for reconstruction, (c) reconstructed RF image using a reweighted conjugate gradient optimization, (d) reconstructed RF image using the Bayesian framework proposed in [20].

\section{Doppler}

CS has also been proposed for Doppler imaging [32, 33]. In [32], the authors applied CS on duplex ultrasonography which is a mode of medical ultrasonography that allows to visualize at the same time the inner structure of the body (B-mode) and the blood flow at a particular point in the body (Doppler mode). This mode requires a strategy for alternating B-mode and flow emissions. Traditional strategies either halve the maximum measurable velocity or introduce gaps in the flow data.
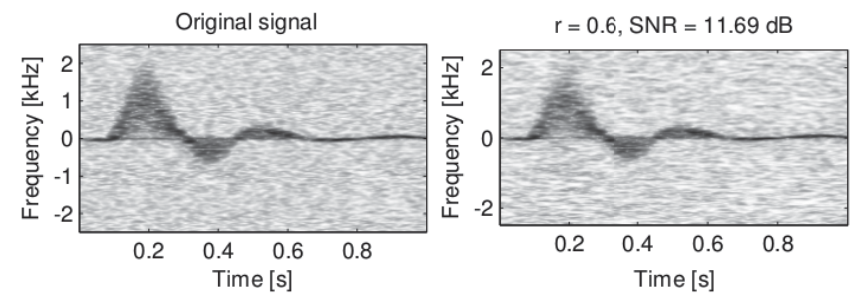

Figure 7: In vivo Doppler result from a femoral artery. a) real sonogram; b) reconstructed sonogram based on CS. $r$ corresponds to the ratio of the number of Doppler samples retained to the total number of samples.

In [32], CS is used to reconstruct the Doppler signal segment by segment. More precisely, the authors made the assumption that the Fourier transform of the Doppler signal is sparse. Using the notation introduced in section II, $\Phi$ stands in this section for the temporal acquisition mask (defining the random positions where temporal Doppler samples are measured), is the inverse Fourier transform, y are the randomly measured Doppler samples and $\mathrm{v}$ is the Fourier transform of the Doppler signal to be reconstructed. One particularity of this work is the decomposition of the signal to be reconstructed $\mathrm{y}$ in several segments of same length. $\ell_{1}$ minimisation is then applied on each segment separately. By doing so, the reconstruction algorithm, which is by far the most consuming step, is applied on a reduced number of samples allowing to reach real-time applicability. The proposed method has been evaluated on both simulated and real sonograms. In both cases, reconstructed Doppler signals with acceptable SNR (higher than $10 \mathrm{~dB}$ ) are obtained using $50 \%$

\section{DisCUSSION AND CONCLUSION}

As presented in this paper, compressed sensing medical ultrasound is a very recent field of research that can lead to drastic modifications in the way ultrasound scanners are developed. The technique is feasible but far from its technological applicability. The key points for CS to work are 1) a sparsifying basis $\Psi$, 2) a measurement basis $\Phi$ incoherent with $\Psi, 3)$ dedicated acquisition material (electronics) and 4) fast and robust reconstruction algorithms. Improvements are necessary for all of these points and compressive sensing medical ultrasound remains an open problem. In particular, efforts should be made in order to maintain the real-time characteristic of medical ultrasound which still makes it a particular imaging modality.

\section{ACKOWLEDGMENT} US-3D

This work has been partially financed by CNRS PEPS CS-

\section{REFERENCES}

[1] E. J. Candes, J. Romberg, and T. Tao, "Robust uncertainty principles: exact signal reconstruction from highly incomplete frequency information," IEEE Transactions on Information Theory, vol. 52, pp. 489-509, 2006.

[2] E. J. Candes and M. B. Wakin, "An Introduction To Compressive Sampling," IEEE Signal Processing Magazine, vol. 25, pp. 21-30, 2008.

[3] D. L. Donoho, "Compressed sensing," IEEE Transactions on Information Theory, vol. 52, pp. 1289-1306, 2006.

[4] M. Lustig, D. Donoho, and J. M. Pauly, "Sparse MRI: The application of compressed sensing for rapid MR imaging," Magnetic Resonance in Medicine, vol. 58, pp. 1182-1195, 2007.

[5] F. J. Herrmann and G. Hennenfent, "Non-parametric seismic data recovery with curvelet frames," Geophysical Journal International, vol. 173, pp. 233248, 2008.

[6] J. A. Jensen, "Spectral velocity estimation in ultrasound using sparse data sets," The Journal of the Acoustical Society of America, vol. 120, pp. 211-220, 2006.

[7] E. Candès and J. Romberg, "Sparsity and incoherence in compressive sampling," Inverse Problems, vol. 23, p. 969, 2007.

[8] J. A. Tropp and A. C. Gilbert, "Signal Recovery From Random Measurements Via Orthogonal Matching Pursuit," IEEE Transactions on Information Theory, vol. 53, pp. 4655-4666, 2007. 
[9] E. J. Candès, "The restricted isometry property and its implications for compressed sensing," Compte Rendus de l'Academie des Sciences, vol. 346, pp. 589-592, 2008.

[10] E. J. Candes and T. Tao, "Decoding by linear programming," IEEE Transactions on Information Theory, vol. 51, pp. 4203-4215, 2005.

[11] A. Austeng and S. Holm, "Sparse 2-D arrays for 3-D phased array imaging - design methods," IEEE Transactions on Ultrasonics, Ferroelectricity and Frequency Control, vol. 49, pp. 1073-1086, 2002.

[12] M. Schiffner, T. Jansen, and G. Schmitz, "Compressed Sensing for Fast Image Acquisition in Pulse-Echo Ultrasound," Biomedical Engineering, vol. 57, pp. 192-195, 2012.

[13] M. Schiffner and G. Schmitz, "Fast Pulse-Echo Ultrasound Imaging Employing Compressive Sensing," in IEEE International Ultrasonics Symposium, Orlando, Florida, USA, 2011, p. in press.

[14] N. Wagner, Y. C. Eldar, A. Feuer, G. Danin, and Z. Friedman, "Xampling in Ultrasound Imaging," in SPIE Medical Imaging, 2011.

[15] N. Wagner, Y. C. Eldar, A. Feuer, and Z. Friedman, "Compressed beamforming applied to B-mode ultrasound imaging," in IEEE International Symposium on Biomedical Imaging (ISBI), Barcelona, Spain, 2012, p. in press.

[16] M. Shen, Q. Zhang, and J. Yang, "A novel receive beamforming approach of ultrasound signals based on distributed compressed sensing," in Instrumentation and Measurement Technology Conference (I2MTC), 2011, pp. 1-5.

[17] Q. Zhang, B. Li, and M. Shen, "A measurementdomain adaptive beamforming approach for ultrasound instrument based on distributed compressed sensing: Initial development," Ultrasonics, p. in press, 2012.

[18] X. Zhuang, Y. Zhao, Z. Dai, H. Wang, and L. Wang, "Ultrasonic signal compressive detection with subNyquist sampling rate," Journal of Scientific and Industrial Research, vol. 71, pp. 195-199, 2012.

[19] J. A. Jensen, "Field: A Program for Simulating Ultrasound Systems," Medical \& Biological Engineering \& Computing, vol. 34, pp. 351-353, 1996.

[20] J. A. Jensen and N. B. Svendsen, "Calculation of pressure fields from arbitrarily shaped, apodized and excited ultrasound transducers," IEEE Transactions on Ultrasonics, Ferroelectrics and Frequency Control, vol. 39, pp. 262-267, March 19921992.

[21] H. Liebgott, R. Prost, and D. Friboulet, "Prebeamformed RF signal reconstruction in medical ultrasound using compressive sensing," Ultrasonics, p. accepted, 2012.

[22] D. Friboulet, H. Liebgott, and R. Prost, "Reconstruction de données RF ultrasonores par compressive sensing," in GRETSI, Bordeaux, France, 2011, p. Accepted.

[23] D. Friboulet, H. Liebgott, and R. Prost, "Compressive sensing for raw RF signals reconstruction in ultrasound," in IEEE International Ultrasonics Symposium, San Diego, California, USA, 2010, pp. 367-370.

[24] H. Shaoyan, Y. Ming, and D. Mingyue, "Compressed Sensing for RF Signal Reconstruction in B-model Ultrasound Imaging," in International Conference on Intelligent Computation and Bio-Medical Instrumentation (ICBMI), 2011, pp. 19-22.

[25] L. Demanet and L. Ying, "Wave atoms and sparsity of oscillatory patterns," Applied and Computational Harmonic Analysis, vol. 23, pp. 368-387, 2007.

[26] C. Quinsac, A. Basarab, and D. Kouamé, "Frequency domain compressive sampling for ultrasound imaging," Advances in Acoustics and Vibration, Advances in Acoustic Sensing, Imaging, and Signal Processing, vol. 12, pp. 1-16, 2012.

[27] C. Quinsac, A. Basarab, J. M. Girault, and D. Kouamé, "Compressed sensing of ultrasound images: sampling of spatial and frequency domains," in IEEE Workshop on Signal Processing Systems, San Francisco, California, USA, 2010, pp. 231-236.

[28] C. Quinsac, A. Basarab, J.-M. Gregoire, and D. Kouamé, "3D compressed sensing ultrasound," in IEEE International Ultrasonics Symposium, San Diego, California, USA, 2010, pp. 363-366.

[29] N. Dobigeon, A. Basarab, D. Kouamé, and J.-Y. Tourneret, "Regularized Bayesian compressed sensing in ultrasound imaging," in European Signal and Image Processing Conference (EUSIPCO), Bucharest, Romania, 2011, p. in press.

[30] C. Quinsac, N. Dobigeon, A. Basarab, D. Kouamé, and J.-Y. Tourneret, "Bayesian compressed sensing in ultrasound imaging " in IEEE International Workshop on Computational Advances in MultiSensor Adaptive Processing (CAMSAP), Puerto Rico, 2011, pp. 101-104.

[31] A. Achim, B. Buxton, G. Tzagkarakis, and P. Tsakalides, "Compressive sensing for ultrasound RF echoes using a-Stable Distributions," in International Conference of the IEEE Engineering in Medicine and Biology Society (EMBC 2010), Buenos Aires (Argentina), 2010, pp. 4304-4307.

[32] J. Richy, H. Liebgott, R. Prost, and D. Friboulet, "Spectral doppler using compressive sensing," in IEEE International Ultrasonics Symposium, Orlando, Fl, USA, 2011.

[33] S. M. S. Zobly and Y. M. Kakah, "Compressed sensing: Doppler ultrasound signal recovery by using non-uniform sampling \& random sampling," in 28th National Radio Science Conference (NRSC), 2011, pp. 1-9. 